\title{
A Chaotic Spread Spectrum System for Underwater Acoustic Communication
}

\author{
Hai-Peng Ren • Chao Bai • Qingju Kong • Murilo S Baptista - Celso \\ Grebogi
}

Received: March 342016 / Accepted: date

\begin{abstract}
Acoustic communication is a key technology to exchange information underwater, which is of great significance to explore marine resources and to marine defense. The underwater acoustic channel is a time-space-frequency varying channel characterized by serious multipath effect, limited frequency band, complex environmental noises and significant doppler frequency shift phenomenon, which makes underwater acoustic communication with low Bit Error Rate (BER) to be a challenging task. A novel chaotic spread spectrum acoustic communication method with low BER is proposed in this paper. A chaotic signal, generated by a
\end{abstract}

\section{Hai-Peng Ren}

Shaanxi Key Laboratory of Complex System Control and Intelligent Information Processing, Xian University of Technology, Xian, 710048, China

E-mail: renhaipeng@xaut.edu.cn

Chao Bai

Shaanxi Key Laboratory of Complex System Control and Intelligent Information Processing, Xian University of Technology, Xian, 710048, China

E-mail: bcxaut@foxmail.com

Qingju Kong

Shaanxi Key Laboratory of Complex System Control and Intelligent Information Processing, Xian University of Technology, Xian, 710048, China

E-mail: 1060680710@qq.com

Murilo S Baptista

Institute for Complex System and Mathematical Biology, University of Aberdeen, King's College, Aberdeen, AB24 3UE, UK

E-mail: murilo.baptista@abdn.ac.uk

Celso Grebogi

Institute for Complex System and Mathematical Biology, University of Aberdeen, King's College, Aberdeen, AB24 3UE, UK

E-mail: grebogi@abdn.ac.uk hybrid dynamical system, is used as a spread spectrum sequence at the transmitter end. At the receiver end, a corresponding chaotic matched filter is used to offset the effect of multipath propagation and noise. The proposed method does not require the complicated equalization and modulation-demodulation technologies that are necessary for conventional acoustic communication. Simulation results show that the proposed method has good anti-interference ability and lower BER as compared to other traditional methods.

Keywords Underwater acoustic communication . Hybrid dynamical system - Chaotic spread spectrum communication · Matched filter · Bit error rate

\section{Introduction}

The ocean occupies two-thirds of the surface of the earth, containing abundant energy and mineral resources. With the increasingly prominent problems such as resource shortage, environment pollution monitoring, national security, defence, and so on, the development and exploration of marine resources becomes the focus of scientific and technological research. Acoustic communication is a key technology to exchange information underwater [1], since acoustic signals, in contrast to optic and radio signals, can propagate in water for long range distances.

From the perspective of communication theory, the ocean/sea and other aquatic environments acts as a physical medium that transforms wave signals, i.e. it defines a communication channel. For communication systems based on acoustic signals, the channel bares the name of underwater acoustic channel. It is a random inhomogeneous medium with slow fading and time diffuse. The underwater acoustic channel is characterized 
by large attenuation, low available bandwidth, multipath propagation, doppler effect and complex environmental noises, serious interferences, among others, which present a major challenge to the implementation of a low BER underwater acoustic communication system.

With the establishment of various kinds of communication networks, the spectrum domain is becoming increasingly occupied and mutual communication interference is becoming more severe. Spread spectrum communication is one of the most used communication protocols due to its ability to provide security and robustness to both noise and interference.

Because of its broadband spectrum, sensitive dependence on initial conditions and a delta-function like autocorrelation function, a chaotic signal is a natural choice for communication applications $[2,3]$. Chaos is combined with spread spectrum technology to form the chaotic spread spectrum communication technology. Conventional spread spectrum communication uses pseudo-noise (PN) codes (such as M sequences, Gold sequences, etc.) as spread spectrum codes. PN codes, generally produced by linear feedback shift registers, have fixed periodical structure, and large spikes can be found in their cross-correlated functions in the case of multipath environments. Due to its aperiodic property, chaotic signals, used as spread spectrum sequences, can improve performance as compared to the traditional PN codes. Chaotic spread spectrum communication is mainly divided into two categories: chaotic binary value spread spectrum (CBSS) and chaotic real value spread spectrum (CRSS).

CBSS research focus mainly on how to construct chaotic spread spectrum sequences with good performance (correlation, balance). In Ref. [4], an optimized selection algorithm to improve the balance and correlation performance of chaotic sequences was proposed and it provided low BER in multi-users chaotic direct sequence code division multiple access (DS-CDMA) communication. References [5] and [6] proposed chaotic spread-spectrum sequences produced by quantifying the multiplication of two independent Tent chaotic sequences [7] or by multiplying two independent Tentmaps after the L-bits quantification procedure. Using the composite chaotic sequences guaranteed a lower BER than using single chaotic sequence under the same signalto-noise ratio (SNR) level. In Refs. [8,9], pseudo chaotic sequences were presented and showed that it outperformed the conventional PN sequences. Reference [10] applied a new piecewise Logistic chaotic map [7] to image spread spectrum communication. The BER is decreased as compared to the traditional Logistic chaotic map and a modified Logistic map. In Ref. [11], a one driving variable adaptive controller for synchronization of a fractional order chaotic system was designed and applied to general spread spectrum communication system. Its circuit experimental results showed the effectiveness of the proposed scheme. Reference [12] proposed a second order Chebyshev map to improve the balance property. Reference [13] generated spread spectrum sequence using three dimensional chaotic system and applied the sequence into a direct sequence code division multiple access (DS-CDMA) system to obtain better performance as compared to the sequences generated by one dimensional chaotic systems. However, it required perfect synchronization between the transmitter and the receiver, something difficult to implement in practice. In practical applications of CBSS, the chaotic sequence might yield periodicity due to precision limitation of the hardware. To deal with such problems, References [14-16] proposed some improved quantization methods. In the traditional PN code spread spectrum communication system, PN code synchronizing capture techniques are used at the decoding stage. Such techniques include parallel capture, phase sequence searching, correlation capture and match filter method [17]. Correlation was also used to obtain synchronizing spread spectrum code in order to decode information $[18,19]$. Reference [20] presented code piece interleaving method to improve code tracking performance in chaotic spread spectrum system. Chaotic spread spectrum technology is also applied in Code Division Multiple Access (CDMA) $[13,21]$, Orthogonal Frequency Division Multiplexing (OFDM) [22], Ultra Wideband (UWB) [23] communication system. Most of the aforementioned methods are dedicated to free space wireless communication channel. When chaotic spread spectrum communication methods are used in underwater acoustic channels, the equalization techniques have to be used to deal with serious distortion caused by the underwater acoustic channel [24], as done for other air-based wireless communication [25-27].

Chaotic Direct Sequence Spread Spectrum (CD3S) communication method, as a kind of CRSS, was proposed by U. Parlitz [28] and C. D. McGillem [29], independently, in 1994. The idea of CD3S was to implement chaotic spread spectrum sequence synchronization as well as to decode the information by filtering techniques, such as Extended Kalman Filter (EKF), Unscented Kalman Filter (UKF) and Particle Filter (PF). Reference [30] proposed two decoding methods for using CD3S in underwater acoustic channel. One of them used Exact Spreading Sequence copy, the other used Dual UKF (DUKF) [31] to estimate the spreading spectrum sequence and the delivered information symbol. The first method relied on the exact repli- 
ca of the spread spectrum sequence, which increased the complexity in practical implementation. The second method had a slightly higher BER because no equalizer had been used. Reference [32] also proposed two decoding methods for CD3S used in acoustic channel. One was similar to the conventional matched filter structure, which consisted of a Delay Lock Loop for code tracking and a Costas Loop for carrier tracking, as usually used in standard Direct Sequence Spread Spectrum (DS/SS) system. The other method used DUKF to estimate the chaotic spread spectrum code, the data symbol, and the carrier phase, simultaneously. The sea trial showed that the first method could get good performance under time-invariant channel, while the second method was fit for complex time variant channel. In [33], a carrier recovery block (e.g., Costas Phase Locked Loop) was used to compensate carrier phase deviation and the DUKF was used to estimate the chaotic spreading code together with the data. Compared with the DUK$\mathrm{F}$ in [32] on the time variant Additive White Gaussian Noise(AWGN) channel and the time-invariant AWGN channel, the method in [33] had better performance and lower BER. The multipath propagation was not explicitly taken into account in [30-33]. In [34], the multipath effect was considered at the Bay of Brest (France), and the sea trial showed that the performance of DUKF degraded in the multipath environment.

A growing interest in attacking chaotic spread spectrum communication has emerged. References [35-38] presented several methods for attacking CD3S communication systems. Reference [35] proposed a single Unscented Kalman Filter (UKF) filtering method to recover the binary information and the chaotic spread code in AWGN channel without any knowledge of the chaotic transmitter. Reference [36] used Interacting Multiple Model-Unscented Kalman Filter (IMM-UKF) to estimate spread spectrum code and binary information in multipath fading and noisy channel, which yields lower BER as compared to the method in [35]. Reference [37] proposed blind equalization and UKF to estimate binary information and chaotic spread code in channel with multipath propagation and white noise. Reference [38] used particle filter (PF) to demodulate CD3S signal in the channel with colored or non-gaussian noise; compared with resilient back propagation neural network algorithm and UKF-based algorithm, PF had lower BER for decoding.

In this paper, based on the fundamental idea in [25], a novel chaotic direct sequence spread spectrum communication for underwater environment is presented and the detailed analysis is given to evaluate the performance of the proposed method. The proposed method has the merits of the CBSS and the CRSS.
The chaotic spreading sequences, generated by the hybrid dynamical system, are continuous signals containing random-like PN discrete codes. The information bits multiply the chaotic sequence without quantization, which avoids the periodicity of the chaotic spreading code induced by quantization [14-16]. Compared with the methods in [30-34], the base frequency of the proposed chaotic spreading code can be conveniently set by changing a parameter in order to adapt to the channel bandwidth. It needs neither to use spectrum shift technology nor frequency modulation, which simplifies the structure of the communication system. By adding synchronous frames into the spreading spectrum signals, the proposed method can demodulate the information without an accurate synchronization between transmitter and receiver and/or code synchronous acquisition, which further reduces the complexity, thus facilitating the implementation. If the authorized receiver knows the rules of adding synchronous frames, it can decode information trivially. In addition, a matched filter is used at the receiver end to reduce the effect of interference and noise. As a result, the proposed method can communicate at low BER in low SNR (signal noise ratio) environment. The comparison results in Sec. 5 show the good performance of the proposed method.

\section{Underwater acoustic channel model}

The geometric model [39] of the underwater acoustic channel is shown in Fig. 1. Assume that $R$ is the distance between transmitter and receiver, $d_{1}$ is the depth of the transmitter, $d_{2}$ is the depth of the receiver, $h$ is the height of the water column.

We assume that the parameter $s$ is the number of reflections with the surface, and $b$ is the number of reflections with the bottom. Assume that $D_{0}$ is the distance of the direct eigenray, $D_{s b}(0 \leq s-b \leq 1)$ is the distance of the eigenray reflected by the surface only, and $D_{b s}(0 \leq b-s \leq 1)$ is the distance of the eigenray reflected by the bottom. We have

$$
\begin{aligned}
& D_{0}=\sqrt{R^{2}+\left(d_{1}-d_{2}\right)^{2}} \\
& D_{s b}=\sqrt{R^{2}+\left[2 b h+d_{1}-(-1)^{s-b} d_{2}\right]^{2}} \\
& D_{b s}=\sqrt{R^{2}+\left[2 b h-d_{1}+(-1)^{b-s} d_{2}\right]^{2}}
\end{aligned}
$$

The transmission loss due to spherical spreading along the eigenray propagation can be written as $L_{S S}(D)$. When sound propagates in the sea, part of the acoustic energy is continuously transformed into heat, denoted as $L_{A}(D)$. The loss caused by the interaction with the 


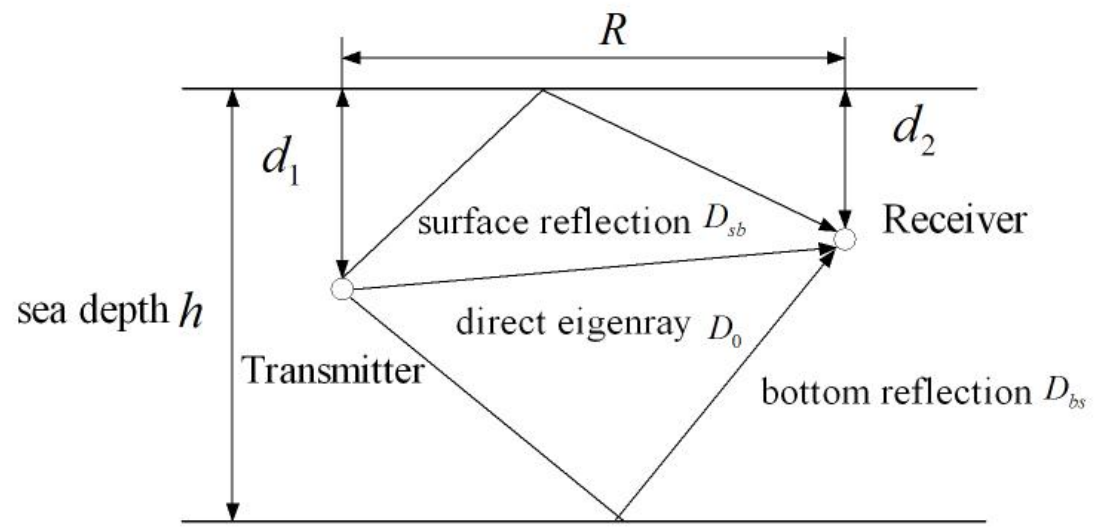

Fig. 1 Geometric model of the warm shallow water acoustic (WSWA) channel.

surface and the loss caused by the interaction with the bottom are denoted as $L_{S}, L_{B}(\theta)$, respectively.

$L_{S S}(D)=\sqrt{\frac{1}{D^{2}}}=\frac{1}{D}$

and

$L_{A}(D)=\exp \left[-0.998 D\left(\frac{S A f_{T} f^{2}}{f_{T}^{2}+f^{2}}+\frac{B f^{2}}{f_{T}}\right)\right]$,

where $A=0.00000234, B=0.00000338, S$ is the salinity and $T$ (Celsius) is the temperature of the seawater, $f$ is the frequency of the transmitted signal, and $f_{T}$ is the relaxation frequency.

$f_{T}=2.19 \times 10^{6-\frac{1520}{T+273}}$,

$L_{S}=-1$,

$L_{B}(\theta)=\left|\frac{m \cos \theta-\sqrt{n^{2}-\sin ^{2} \theta}}{m \cos \theta+\sqrt{n^{2}-\sin ^{2} \theta}}\right|$,

where

$m=\frac{\rho_{1}}{\rho}, n=\frac{c}{c_{1}}$.

The parameters $\rho, \rho_{1}$ are the density of seawater and seabed, respectively. The parameters $c, c_{1}$ are the sound speed in seawater and seabed, respectively. Let angle $\theta_{s b}$ correspond to an eigenray $D_{s b}$ and angle $\theta_{b s}$ correspond to an eigenray $D_{b s}$. Then, we have

$\theta_{s b}=\tan ^{-1}\left(\frac{R}{2 b h+d_{1}-(-1)^{s-b} d_{2}}\right)$

and

$\theta_{b s}=\tan ^{-1}\left(\frac{R}{2 b h-d_{1}+(-1)^{b-s} d_{2}}\right)$
We consider the arrival time-lag of multipath eigenrays. Let $\tau_{1}$ be the arrival time-lag of eigenray $D_{s b}$, and $\tau_{2}$ be the arrival time-lag of eigenray $D_{b s}$. We have

$\tau_{1}=\frac{D_{s b}-D_{0}}{c}$

$=\frac{2}{R c}\left[b^{2} h^{2}+b h d_{1}-(-1)^{s-b} b h d_{2}+(s-b) d_{1} d_{2}\right]$

$\tau_{2}=\frac{D_{b s}-D_{0}}{c}$

$\approx \frac{2}{R c}\left[b^{2} h^{2}-b h d_{1}+(-1)^{b-s} b h d_{2}+(b-s) d_{1} d_{2}\right]$.

As seen from Eq. (12), the time delay of the eigenray depends on the horizental distance between the transmitter and the receiver, $R$, the sound speed, $c$, the depth of the transmitter, $d_{1}$, and the depth of the receiver, $d_{2}$, all this dependent variables are time variant. To consider all time-variant factors, the time delay changes are expressed by

$J_{1}=\frac{\partial \tau_{1}}{\partial R} \Delta R+\frac{\partial \tau_{1}}{\partial c} \Delta c+\frac{\partial \tau_{1}}{\partial d_{1}} \Delta d_{1}+\frac{\partial \tau_{1}}{\partial d_{2}} \Delta d_{2}$,

$J_{2}=\frac{\partial \tau_{2}}{\partial R} \Delta R+\frac{\partial \tau_{1}}{\partial c} \Delta c+\frac{\partial \tau_{2}}{\partial d_{1}} \Delta d_{1}+\frac{\partial \tau_{2}}{\partial d_{2}} \Delta d_{2}$.

where $\Delta R$ is equivalent distance variation, we assume $R \gg d_{1}, R \gg d_{2}$, and $R \gg h$, then the derivative with respect to $R$ is close to $0 . \Delta d_{1}$ and $\Delta d_{2}$ are the depth variations of the transmitter and the receiver, respectively. $\Delta c$ is the sound speed variation. The point to be noticed here is that the effect of variant $c$ is small. These variations are treated together as Gaussian random variables with different variances [39]:

$\sigma_{s b}^{2}=\frac{4 \sigma_{d}^{2}}{R^{2} c^{2}}\left[2 b^{2} h^{2}+(s-b)\left(d_{1}^{2}+d_{2}^{2}+2 b h d_{1}+2 b h d_{2}\right)\right]$,

$\sigma_{b s}^{2}=\frac{4 \sigma_{d}^{2}}{R^{2} c^{2}}\left[2 b^{2} h^{2}+(b-s)\left(d_{1}^{2}+d_{2}^{2}-2 b h d_{1}-2 b h d_{2}\right)\right]$,

where $\sigma_{d}$ is the Root-Mean-Square (RMS) equivalent relative movement from the transducer to the receiver.

The values $A_{j}(t)$ ( $j=1,2,3$, the number of eigenrays), representing the time-varying feature of the amplitude changes of each eigenray, are modeled as independent Rayleigh processes. The noise in the model is considered as Gaussian white noise for simplicity, which is denoted 
as $n(t)$. Assume that the transmitting signal is $x(t)$ and the receiving signal is $y(t)$. Therefore, the mathematical model of the underwater acoustic channel can finally be given as

$y(t)=\alpha_{1} x(t)+\sum_{j=1}^{N-1} \alpha_{j} x\left(t-\left(\tau_{j}+J_{j}(t)\right)\right)+n(t)$.

where $\alpha_{1}=A_{1}(t) L_{S S}\left(D_{0}\right) L_{A}\left(D_{0}\right), \alpha_{j}=A_{j}(t) L_{S S}\left(D_{M}\right)$ $L_{A}\left(D_{M}\right)\left(L_{S}\right)^{s}\left(L_{B}\left(\theta_{M}\right)\right)^{b}$, and $N=6$ is the number of multiple paths, $M=s b(0 \leq s-b \leq 1)$ or $b s(0 \leq b$ $s \leq 1)$. The parameters of the acoustic channel used in this paper are listed in TABLE 1.

The characteristics of the underwater acoustic channel require that the communication system to resist multipath propagation, time-varying characteristics, limited frequency band, complex ambient noises, interference and significant Doppler frequency shift phenomenon as compared to the free space wireless communication counterpart.

\section{Spread spectrum communication method using chaos generated by hybrid system}

\subsection{The hybrid dynamical system}

We consider the hybrid dynamical system, comprised of a continuous state $u(t) \in R$ and a discrete state $s_{i} \in\{ \pm 1\}$ [40], given by

$\ddot{u}-2 \beta \dot{u}+\left(\omega^{2}+\beta^{2}\right)(u-s)=0$.

The discrete dynamics is defined by the guard condition. $s$ only switches at time $\dot{u}=0$ and remains unchanged in the rest of the time,

$s=\operatorname{sgn}(u(t))=\left\{\begin{array}{l}1, u(t) \geq 0 \\ -1, u(t)<0\end{array}\right.$

Here $\omega=2 \pi f, \beta=f \ln 2$, and integer $f$ is the base frequency of the signal. $f$ is used to adjust the signal bandwidth to fit the communication channel; For a fixed $f$, parameter $\beta$ determines the Lyapunov exponent of the system. For the system to have more complex trajectory, $\beta$ should approach $f \ln 2$. Switching period of the discrete state $s$ is denoted as $T_{c}=\frac{2 \pi}{\omega}=\frac{1}{f}$. The chaotic signal waveform of the hybrid dynamical system for $f=1 \mathrm{~Hz}$ are shown in Fig. 2(a), and we know that the largest Lyapunov exponent of the hybrid system, calculated from time series, is positive: 0.695. Figure 2(b) shows the autocorrelation of the state variable of the hybrid system for $f=45 \mathrm{kHz}$. It indicates that the hybrid system has a pulse type autocorrelation function. As can be seen from Fig. 2, the signal, generated by the

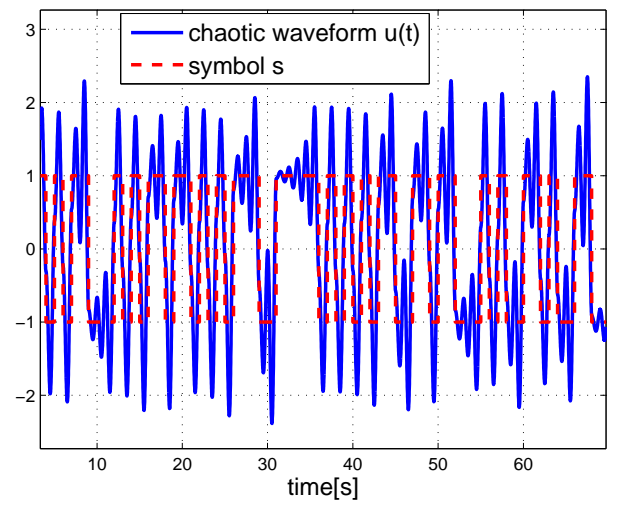

(a)

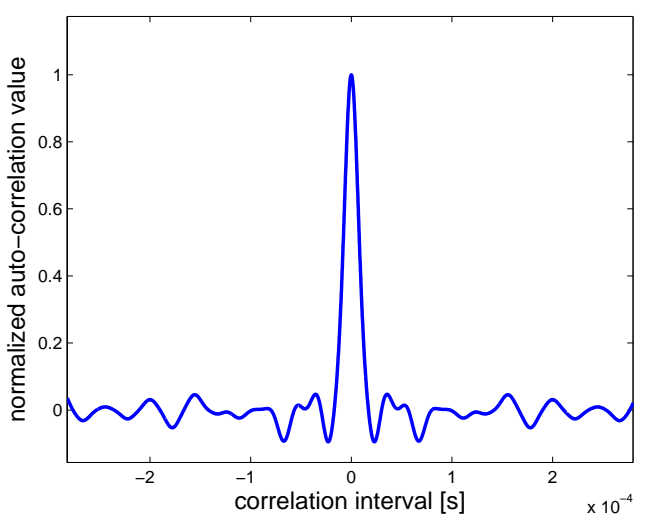

(b)

Fig. 2 The simulations of the hybrid dynamical system. (a) Time domain chaotic waveform generated by the hybrid dynamical system. (b) Auto-correlation function of the signal of the hybrid dynamical system $(f=45 \mathrm{kHz})$.

hybrid dynamical system, is chaotic. The binary representation of $s$ is $s_{i}$, where $i=1,2, \ldots, l$, and $l$ is the number of times that $s$ has changed its sign.

3.2 The matched filter corresponding to the chaotic signal generated by the hybrid system

The matched filter [40] corresponding to the hybrid system (16) that can functionally filter out noise is given by

$\left\{\begin{array}{l}\ddot{u}_{4}(t)+2 \beta \dot{u}_{4}(t)+\left(\omega^{2}+\beta^{2}\right)\left(u_{4}(t)-\eta(t)\right)=0 \\ \dot{\eta}(t)=u_{3}\left(t+T_{c}\right)-u_{3}(t)\end{array}\right.$

where $u_{3}(t)=y(t)$ is the received signal after being transmitted through the underwater acoustic channel, representing the filter input, and $u_{4}(t)$ is the filter output. The definition of $\omega$ and $\beta$ are the same as that in Eq. (16). In this work, we show that this filter can also successfully resolve the interference caused by the multipath propagation. 
Table 1 Parameters in WSWA channel

\begin{tabular}{|c|c|c|c|}
\hline parameter name & parameter value & parameter name & parameter value \\
\hline sea depth $h$ & $100 m$ & seabed sound speed $c_{1}$ & $1650 \mathrm{~m} / \mathrm{s}$ \\
\hline temperature of seawater $T$ & $20^{\circ} \mathrm{C}$ & seawater density $\rho$ & $1023 \mathrm{~kg} / \mathrm{m}^{3}$ \\
\hline transmitter depth $d_{1}$ & $30 m$ & seabed density $\rho_{1}$ & $1500 \mathrm{~kg} / \mathrm{m}^{3}$ \\
\hline receiver depth $d_{2}$ & $50 m$ & seawater salinity $S$ & $35 p p t$ \\
\hline propagation distance $R$ & $1200 m$ & transducer RMS movement $\sigma_{d}$ & $0.25 m$ \\
\hline seawater sound speed $c$ & $1539 \mathrm{~m} / \mathrm{s}$ & number of multiple paths & 6 \\
\hline 1 surface/bottom reflections $s b$ & 21 & 4 surface/bottom reflections $s b$ & 45 \\
\hline 2 surface/bottom reflections $s b$ & 32 & 5 surface/bottom reflections $s b$ & 23 \\
\hline 3 surface/bottom reflections $s b$ & 43 & & \\
\hline
\end{tabular}

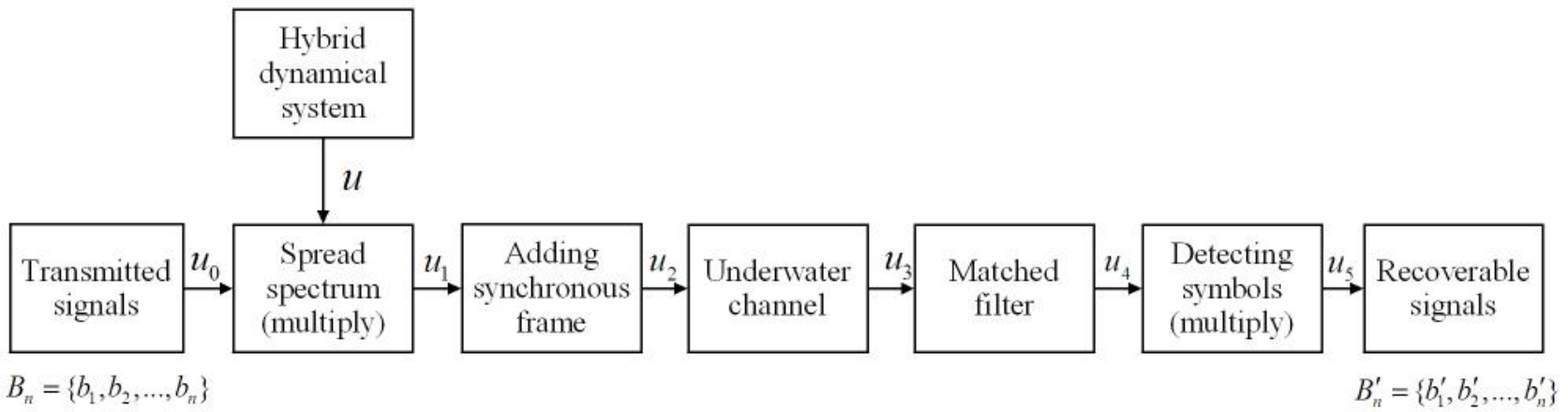

Fig. 3 Chaotic spread spectrum communication system block diagram.

3.3 The proposed chaotic spread spectrum communication method

The block diagram of the proposed communication method is shown in Fig. 3. The spread spectrum signal $u_{1}$ is obtained by multiplying the chaotic spread spectrum sequence $u$, produced by the hybrid system, and the rectangular waveform $u_{0}$ corresponding to transmitted bits, $B_{n}=\left\{b_{1}, b_{2}, \ldots, b_{n}\right\}$ ( $b_{n}$ is the $n t h$ binary information). Then, the synchronous frame is incorporated into $u_{1}$ to finalize the transmitted signal $u_{2}$. The synchronous frame is used to facilitate the decoding of the binary information at the receiver end, and this operation replaces the conventional PN code (spread spectrum sequence) synchronizing capture technique in order to simplify the communication system structure and to relieve the complexity of the algorithm. The received signal after the acoustic channel transmission, $u_{3}(t)$, is fed into the match filter. The filter output, $u_{4}(t)$, is used to detect the symbol at the predefined time. Finally, the synchronous symbol is used to decode the received binary information $B_{n}{ }^{\prime}$. A more detailed description of this protocol will be given as follows.
Assume that $R_{b} b i t / s$, corresponding to code period $T_{b}$, represents the information transmission rate, and $R_{c}$ bit/s represents the symbol generating rate of the hybrid system. Therefore, the spreading gain is denoted as $L=\frac{R_{c}}{R_{b}}=\frac{1 / T_{c}}{1 / T_{b}}=\frac{T_{b}}{T_{c}}$. This means that the spectrum of the transmitted signal is enhanced by $L$ times as compared to the original signal spectrum. Figure 4 illustrates our proposed chaotic spread spectrum communication method for the parameter $f=8 \mathrm{~Hz}$ in the hybrid system. Figure 4(a) shows the rectangular waveform $u_{0}$ corresponding to two binary bits $B_{2}=\{1,-1\}$ to be transmitted, where $T_{b}=0.5 \mathrm{~s}$. The carrier waveform (or spread spectrum sequence) generated by the hybrid system is shown by the solid line in Fig. 4(b), where $L=\frac{T_{b}}{T_{c}}=4$ is the spread spectrum gain. The carrier waveform, multiplied by $u_{0}$ in Fig. 4(a), forms the spread spectrum signal as shown by the solid line in Fig. 4(c). As shown in Figs. 4 (b) and (c), the continuous chaotic signal is used as a spread sequence; the continuous signal embodies the discrete symbols, given by the hybrid system, which are shown by the dotted lines in Figs. 4(b) and (c). The point to be noticed here is that the chaotic spread spectrum strategy has features of 


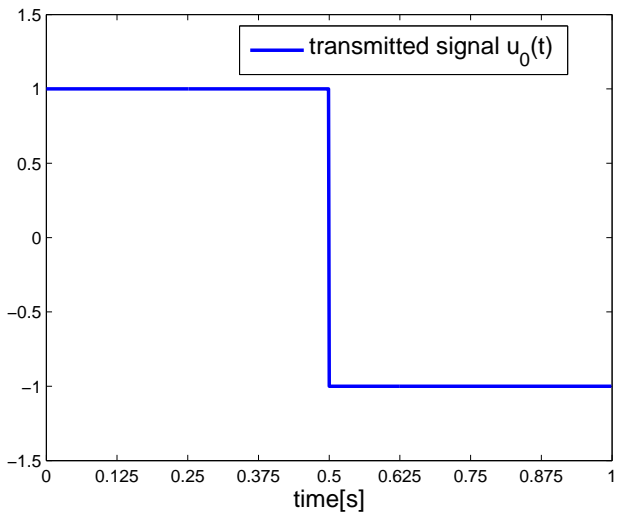

(a)

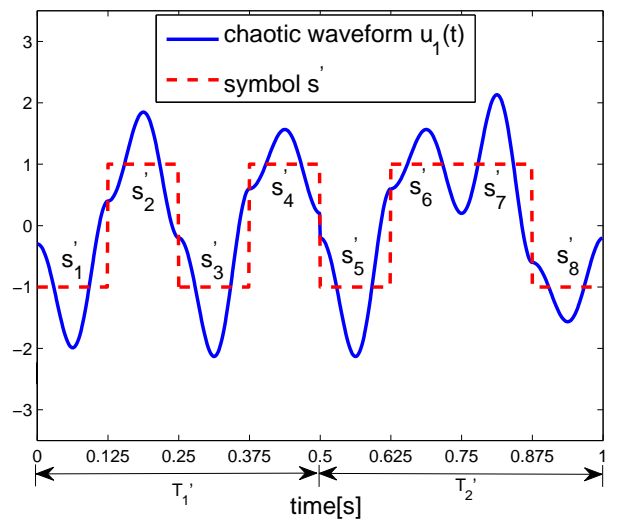

(c)

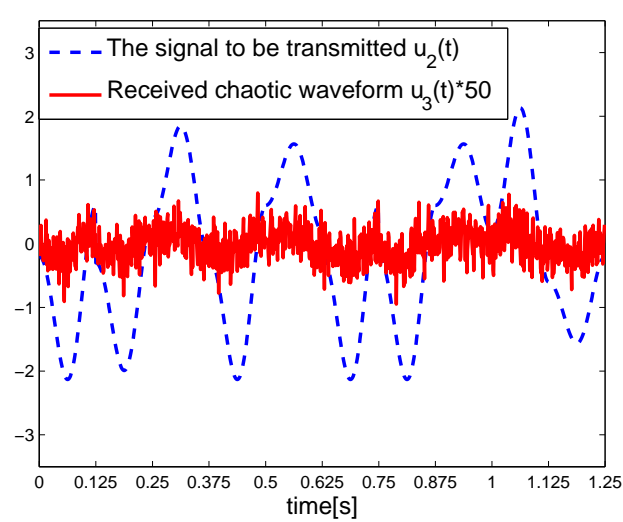

(e)

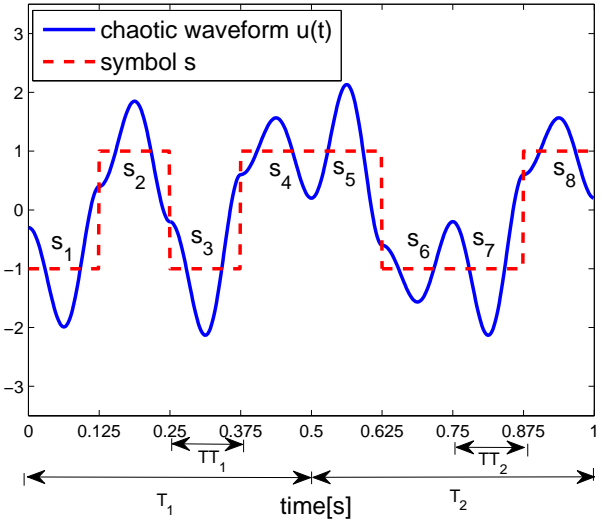

(b)

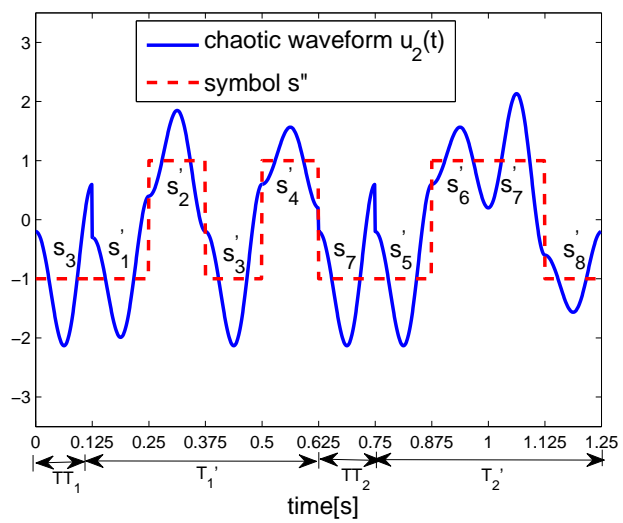

(d)

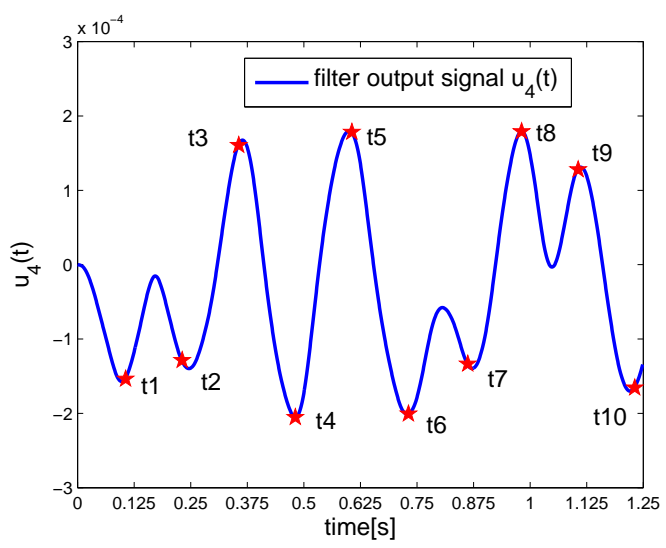

(f)

Fig. 4 Chaotic spread spectrum communication principle. (a) Signal to be transmitted. (b) Chaotic spread spectrum sequence. (c) Signal after spread spectrum. (d) Chaotic spread spectrum signal including synchronous frames. (e) Received waveform. (f) Filter output signal. 
both CRSS and CBSS. The spread spectrum signal, $u_{1}$, is incorporated with the synchronous frame, which is a part of $u$. The synchronous frame could be selected as any segment of $u$ corresponding to one discrete symbol $s_{i}$, within the time interval corresponding to a binary information bit $\left(T_{1}\right.$ and $T_{2}$ in Fig. 4(b)). The synchronization frame, for example, the $u(t)$ signal for the time interval $T T_{1}$, can be inserted to the spread signal $u_{1}$ at the beginning of the period of the binary information signal as shown by Fig. 5. As an illustration, the segment corresponding to the third discrete symbol of the hybrid system ( $s_{3}$ and $s_{7}$ in this example) are selected as the synchronous frames, as indicated by the periods $T T_{1}$ and $T T_{2}$ in Fig. 4(b), and they are inserted before the spread spectrum signal corresponding to every binary information, as shown in Fig. 4(d). We can see from Fig. 4(d) that symbol sequence $s^{\prime \prime}$ consists of synchronous frame symbol, $s$, and the spread spectrum signal symbol, $s^{\prime}$, i.e., $s^{\prime \prime}=\left[s_{3}, s_{1}^{\prime}, s_{2}^{\prime}, s_{3}^{\prime}, s_{4}^{\prime}, s_{7}, s_{5}^{\prime}, s_{6}^{\prime}, s_{7}^{\prime}, s_{8}^{\prime}\right]$. It means that if the symbol in the synchronous frame is the same as the corresponding symbol bit in spread spectrum sequence, for example, $s_{3}=s_{3}^{\prime}$, then the transmitted information is 1 , else, for another example, $s_{7} \neq s_{7}^{\prime}$, the transmitted information is -1 . The synchronous frame selection rule is flexible for each bit, provided that the receiver knows the rule, which helps to increase the security of the communication. By incorporating the synchronous frame to $u_{1}$, we get the signal to be transmitted, denoted as $u_{2}$. After $u_{2}$ is transmitted through the underwater acoustic channel (whose parameters are given in Table 1), the received signal, $u_{3}$, is shown as the solid line in Fig. 4(e). In Fig. $4(\mathrm{e})$, the received signal is magnified 50 times for easy comparison, and the signal to be transmitted is given as the dashed line for comparison purpose. The tremendous distortion to the transmitted signal made by the channel can be seen from Fig. 4(e). The received signal is fed into the match filter, and the output of the filter is denoted as $u_{4}$ shown in Fig. 4(f). The information symbols carried in $u_{4}$ can be detected by

$\sigma_{n}=\operatorname{sign}\left(u_{4}\left(n T_{c}-\Delta t\right)\right)$,

where $\Delta t$ is the detection time delay. Here, $\Delta t$ is set, say, at a fifteen percent of $T_{c}, t_{1}=T_{c}-\Delta t=0.1063 \mathrm{~s}$, $t_{4}=4 T_{c}-\Delta t=0.4813 \mathrm{~s}$, the detection points on $u_{4}$ are marked with pentagrams in Fig. 4(f). We can see that $\sigma_{1}=-1, \sigma_{4}=-1, \sigma_{6}=-1, \sigma_{9}=1$, respectively. By simple multiplication operation, we have $b_{1}{ }^{\prime}=\sigma_{1} * \sigma_{4}=1$ and $b_{2}{ }^{\prime}=\sigma_{6} * \sigma_{9}=-1$, and corresponding to rectangular waveform $u_{5}$ which is the same as $u_{0}$ given by Fig. 4(a). To this end, the transmitted binary information is decoded correctly.

In the following sections, we will analyze the performance of the proposed method.

\begin{tabular}{|c|c|c|c|}
\hline $\begin{array}{c}\text { Synchronous } \\
\text { frame }\end{array}$ & $\begin{array}{c}\text { Spread spectrum } \\
\text { signal }\end{array}$ & $\begin{array}{c}\text { Synchronous } \\
\text { frame }\end{array}$ & $\begin{array}{c}\text { Spread spectrum } \\
\text { signal }\end{array}$ \\
\hline
\end{tabular}

Adding the synchronous frames before the spread spectrum signal

Fig. 5 Form of transmitted signal.

\section{Spectrum analysis of the proposed method}

In this section, we analyze the signal spectrum of the proposed communication scheme. Assume that the information transmission bit rate $R_{b}=100 \mathrm{bit} / \mathrm{s}$, the transmitter parameter $f=10 \mathrm{kHz}$, then $T_{c}=0.1 \mathrm{~ms}$, symbol generation rate of the hybrid system $R_{c}=10 \mathrm{kbit} / \mathrm{s}$, and thus, spectrum spreading gain $L=100$. The frequency spectrum of the original signal is given in Fig. 6(a); it can be calculated that the bandwidth of the signal is approximately $180 \mathrm{~Hz}$. The frequency spectrum of the transmitted signal, after the spread spectrum operation using the proposed method, is shown in Fig. 6(b). It can be calculated that the bandwidth of the transmitted signal is approximately $18.4 \mathrm{kHz}$, which is close to the theoretical value being equal to $180 \mathrm{~Hz} \times 100=18 \mathrm{kHz}$.

The received signal spectrum with signal to noise ratio equal to $-10 \mathrm{~dB}$ is given in Fig. 6(c). It can be seen from Fig. 6(c) that the signal spectrum is almost inundated by the noise spectrum. This received signal is fed into the matched filter, and the filtered signal spectrum is given in Fig. 6(d). It can be seen from Fig. 6(d) that the frequency spectrum of the filtered signal is similar to that of the transmitted one. It can be calculated that the filtered signal bandwidth is about $17.6 \mathrm{kHz}$. It is close to the bandwidth of the signal after spread spectrum.

\section{Performance analysis of the proposed method}

\subsection{Performance analysis in the AWGN channel}

The proposed method is more robust to noise in the AWGN channel compared with conventional spread spectrum communication, due to the usage of the corresponding match filter at the receiver end. Figure 7 (a) shows the BER curves of our proposed communication system and the method in Ref. [24], which generate the spread spectrum sequence by general logistic map SSS1 in Ref. [30], improved logistic mapping SSS2 in [9], the complex spread spectrum code SSS3 in Ref. [5], the piecewise logistic map SSS4 in Ref. [10], and use the conventional PN codes, respectively. In Fig.7(a), the 


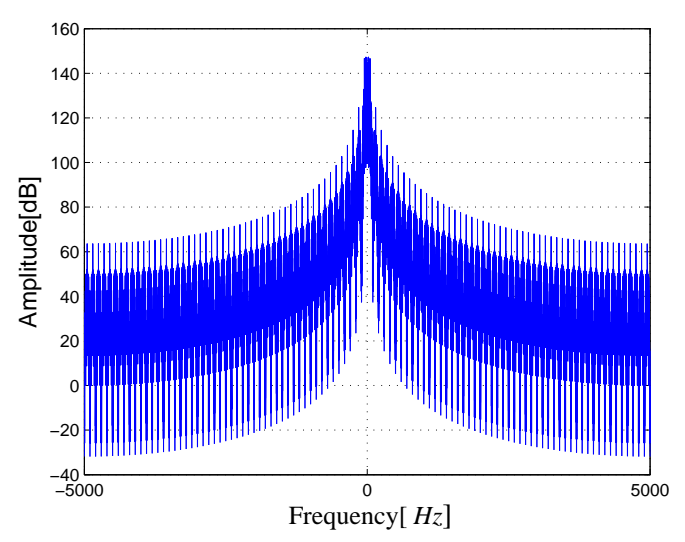

(a)

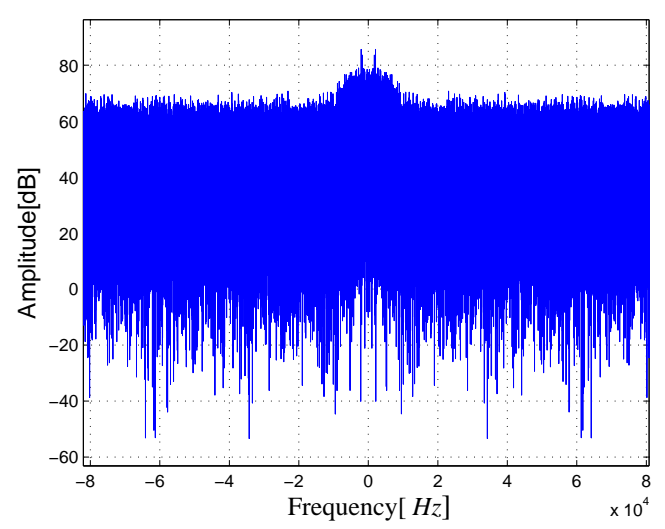

(c)

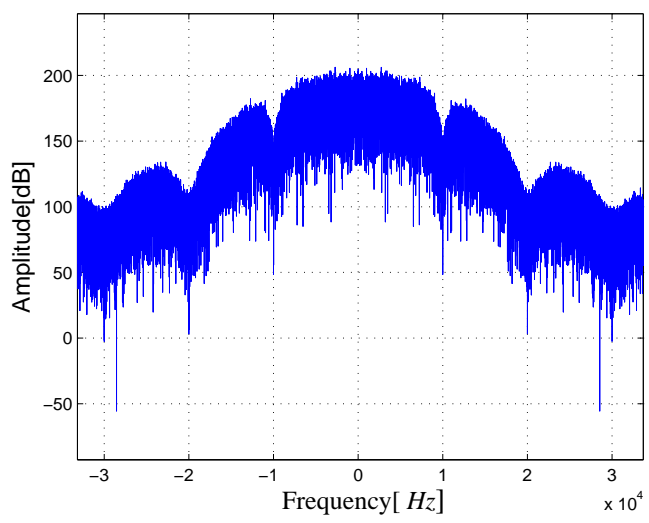

(b)

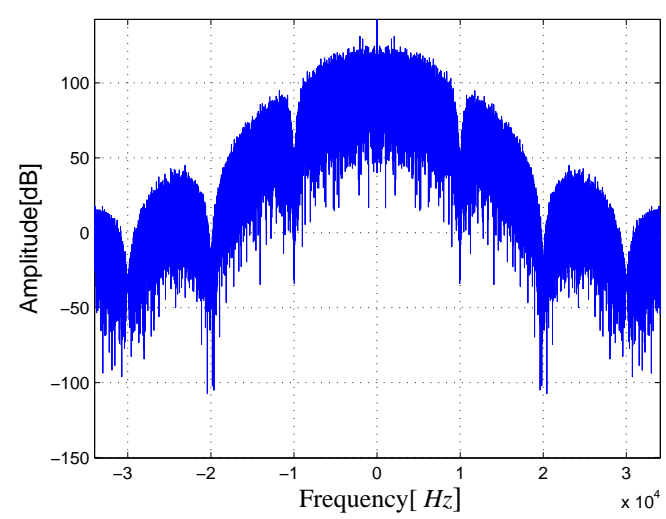

(d)

Fig. 6 The spectrum analysis of chaotic spread spectrum communication method. (a) Spectrum of transmitted binary information. (b) Spectrum of transmitted chaotic spread spectrum signal. (c) Spectrum of received signal. (d) Spectrum of filter output signal.

BER performances are evaluated by 5000 Monte-Carlo simulations with spreading gain $L=10, f=10 \mathrm{kHz}$, and we find that the proposed method yields a very low BER even though the received signal is submerged in noise when SNR is low.

\subsection{Performance analysis in the Rayleigh Fading channel.}

In this subsection, we consider a Rayleigh fading channel to test the BER performances of the proposed method and the conventional spread spectrum communication. The channel model based on a direct ray with Rice distribution and two reflected rays with Rayleigh fading, whose time delays are $6 \mathrm{e}-6 \mathrm{~s}$ and $11 \mathrm{e}-6 \mathrm{~s}$, and the fading gains are $-3 \mathrm{~dB}$ and $-6 \mathrm{~dB}$. We obtained the BER versus SNR with spreading gain $L=10, f=10 k H z$ as shown in Fig. 7(b), and we find that the conventional methods have higher BERs in the Rayleigh Fading channel. Our proposed method still gets better reliability compared to the conventional PN methods and the conventional chaotic spread spectrum method using different chaotic maps.

5.3 Ability to resist to jamming signal in the underwater acoustic channel

To test the ability to resist to single frequency interference (jamming) signal in the acoustic channel in Table 1 , we consider the fixed amplitude and frequency of a sinusoidal interference. Here the chaotic signal base frequency is $f=10 \mathrm{kHz}, \mathrm{SNR}=-5 \mathrm{~dB}$, the amplitude of sinusoidal signal is $A=3$, and the frequency of interference is $10 \mathrm{kHz}$. The sinusoidal interference is added to the transmitted signal $u_{2}$. The transmitted signal, $u_{2}$, the jamming signal, and the received signal with interference, $u_{3}$, are given by the dotted line, the dot dash line and the solid line in Fig. 8(a), respectively. The filter output and the transmitted signal are given by the 


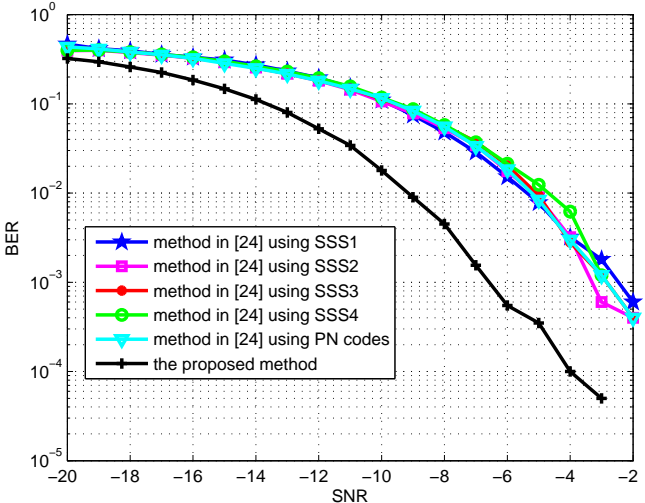

(a)

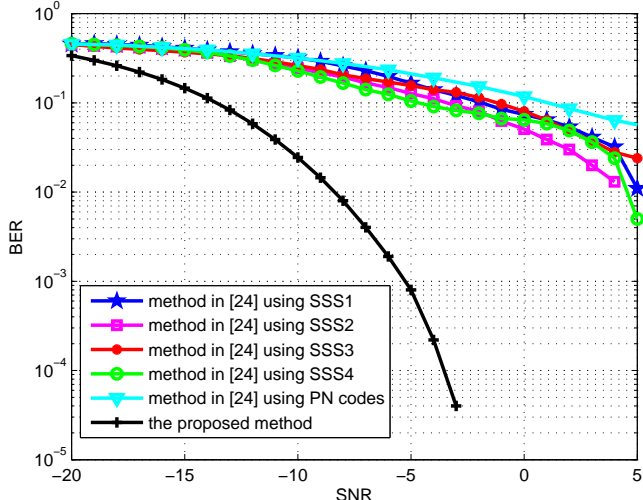

(b)

Fig. 7 (a) BER performances of the chaotic spread spectrum system in the AWGN channel. (b) BER performances of the chaotic spread spectrum system in the Rayleigh Fading channel.

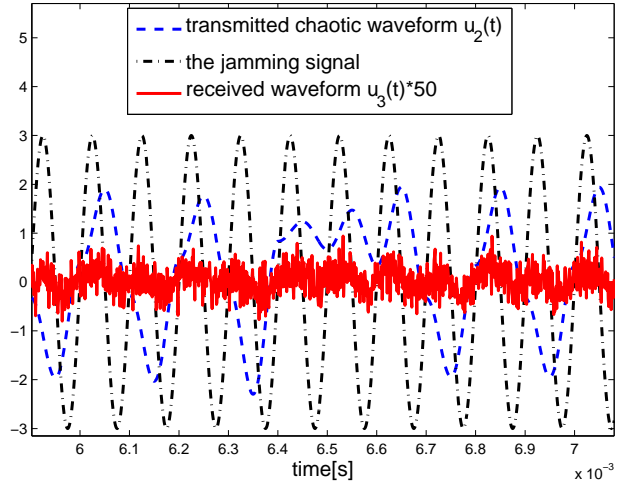

(a)

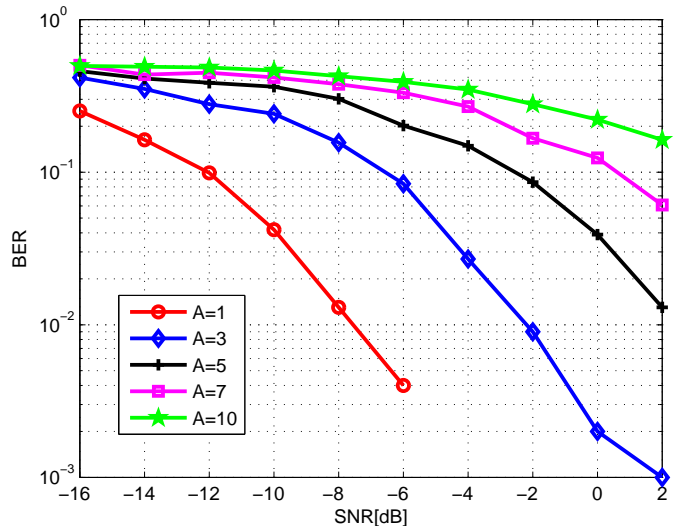

(c)

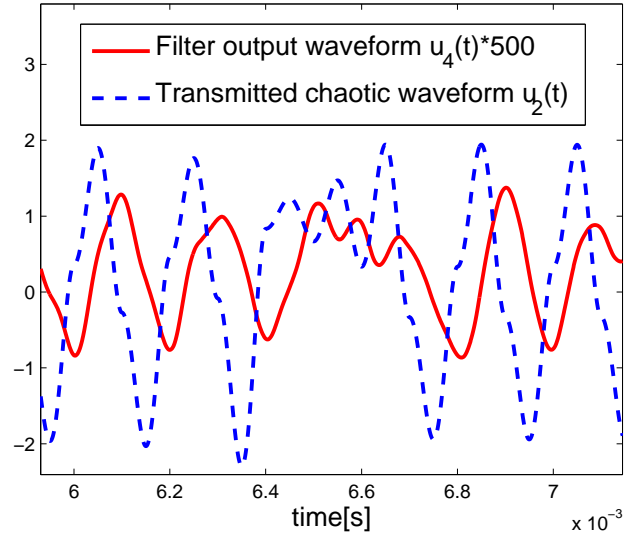

(b)

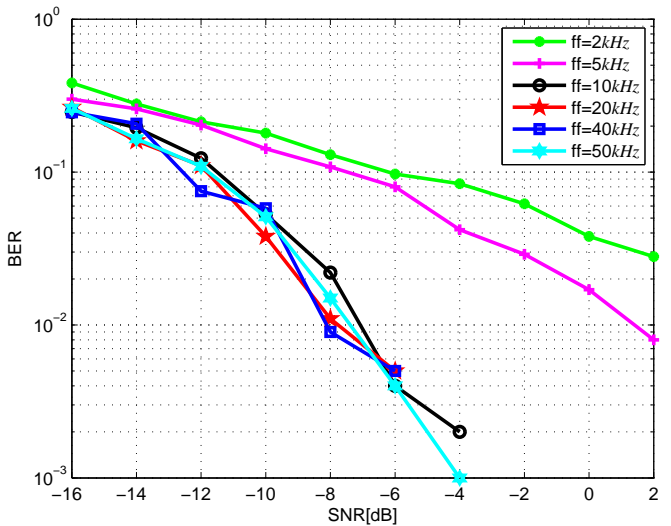

(d)

Fig. 8 The ability to resist to single frequency interference for the proposed chaotic spread spectrum communication method. (a) Comparison of transmitted, jamming and received waveform. (b) Comparison of filter output and transmitted waveform. (c) BER of sinusoidal disturbance signal with different amplitudes. (d) BER of sinusoidal disturbance signal with different frequencies. 


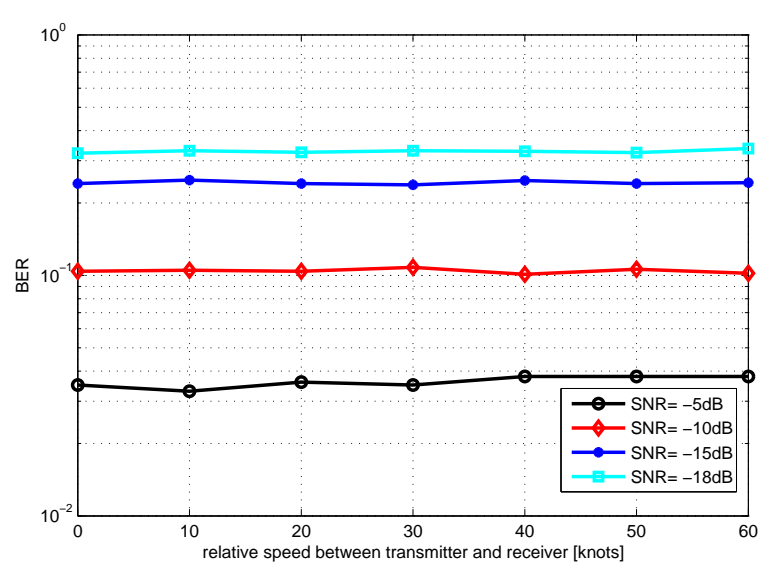

Fig. 9 BER of the proposed method with different relative speeds between the transmitter and the receiver.

solid line and the dotted line in Fig. 8(b), respectively. From Fig. 8(b), we learn that the filter output can approximately reveal the transmitted signal due to the special match filter. The investigation about the effect$\mathrm{s}$ of the amplitude and the frequency of the sinusoidal interference signal versus SNR yields the results shown in Figs. 8(c) and (d), respectively. Figure 8(c) shows the BER variation versus the SNR with different interference amplitudes, where the frequency of the hybrid system and interference signal is fixed at $10 \mathrm{kHz}$, respectively. From Fig. 8(c), we see that BER increases with the increasing of the interference amplitude. Figure $8(\mathrm{~d})$ shows the BER variation versus SNR with different interference frequencies, $\mathrm{ff}$, where the amplitude of the interference is fixed at 1. From Fig. 8(d), we obtain that the BER increases with the decreasing of the interference frequency when the interference frequency is lower than the base frequency of the the hybrid system. When the frequency of the interference is larger than the base frequency, the BER curves are about the same. This means that, when the interference frequency is larger than the base frequency, it does not affect the BER in general.

\subsection{Doppler effect analysis}

In the acoustic channel, the Doppler effect is mainly caused by the relative motion between the transmitter and the receiver. Due to the low acoustic speed, the Doppler effect in the acoustic communication is more serious, as compared to its air-based counterpart. In this subsection, we investigate the Doppler effect on our proposed communication scheme.
In the frequency domain, Doppler effect makes the frequency to change according to the relative velocity. The frequency shift can be given as

$f_{d}=\frac{v}{c} f \cos \theta$

where $v(\mathrm{~m} / \mathrm{s})$ represents the relative velocity between the transmitter and receiver, $c$ represents sound speed in the underwater channel, $f$ represents the frequency of the signal transmitted in the underwater communication channel, and $\theta$ represents the angle between the relative velocity direction and the sound propagation direction. The Doppler effect makes the frequency of the signal to increase, $f^{\prime}=f+f_{d}$, if the transmitter and the receiver approach each other, or the frequency of the signal decreases, $f^{\prime}=f-f_{d}$, if the transmitter and the receiver move apart.

In our proposed communication scheme, the Doppler effect makes the period $T_{c}$ to change, e.g., $T_{c}$ becomes smaller as $T_{c}=\frac{1}{f+f_{d}}$, if the transmitter and the receiver approach each other [different ships or underwater vehicles have different speeds]. Here, we assume that the largest relative velocity is 60 knots. Theoretically, the higher is the base frequency of the chaotic system, the worse is the frequency shift caused by the Doppler effect. We consider the base frequency of the chaotic system to be $50 k H z$, which is a very high value for underwater communication in view of the limited bandwidth of the channel. In this relatively worse case scenario, $T_{c}=20$ microsecond $(u s), f_{d} \approx 1.0029 \mathrm{kHz}$, $T_{c}{ }^{\prime}=\frac{1}{f+f_{d}}=19.6067 u s$. We can see that the period variation caused by Doppler effect $\delta t=0.3933 \mathrm{us}$ is only 1.967 percent of $T_{c}$. The simulation results for different relative speeds are shown in Fig. 9. We can see, from Fig. 9, that the BER of the proposed communication method has no significant increase with the increasing of the relative speed. This indicates that the proposed communication method has good Doppler frequency shift resistance performance. As we know, in the conventional sinusoidal carrier waveform communication system, the Doppler frequency shift causes the central frequency of the carrier wave to vary. This variation has to be compensated by using complex algorithms in conventional communication systems in order to decrease BER. As compared to those methods, the proposed communication scheme has better performance in resisting against Doppler frequency shift.

\subsection{Decoding the received signal using filter technique}

As it has been described in the Introduction, in the conventional CD3S communication scheme, the received signal is decoded using a filter technique, without the 
need to know the structure of the transmitter, which is a security weak point of CD3S communication system. Our proposed method avoids this weak point, we will describe our testing in this subsection.

In the proposed communication system, the signal transmitted in the communication channel is chaotic, which has noise-like spectrum and low detectability. The key point of the security of the proposed method lies in the synchronization frame selection rule (including the information of position and length), which is flexible for each bit. Though the eavesdropper can recover the dynamical structure, it will not help to recover the whole waveform. Even if the eavesdropper would know the waveform, if the eavesdropper would not know the rule of the synchronous frame selection, it cannot help for information decoding, where we assume the selection rule of the synchronous frame to be a random choice, i.e., at each frame, one chooses a different interval for the synchronization frame. From this viewpoint, it is secure to use more bits of the digital signal to transfer one information bit.

Recent work in Ref. [35] has proposed a method to estimate the transmitted binary information through the received signal at the receiver end. To test the effectiveness of this filter-based decoding method against our proposed method, the matched filter output, $u_{4}$, is used as the observation signal for the state space estimation model in Ref. [35]. The initial value of the binary information bit is $d(0)=0$ for the method in Ref. [35], and the initial value of hybrid chaotic system is $u(0)=0.36$. The simulation results are given in Fig. 10. The dotted line in Fig. 10(a) gives the actual chaotic spread spectrum sequence, while, the solid line in Fig. 10(a) gives the estimated sequence using the model given by the method in [35]. It can be seen from Fig. 10(a) that the estimation using the method in Ref. [35] is quite different from the actual one. Figure 10(b) gives the estimated binary information using the method in Ref. [35] and the actual transmitted information. We conclude from the results in Fig. 10 that half of the estimated information is wrong. Therefore, the method in Ref. [35] can not decode our proposed communication scheme. The other existing methods to break the CD3S system in Refs. [36-38] are based on the model in Ref. [35]; therefore, they are unable to decode the information of our proposed scheme from the received signal as well.

\subsection{Performances comparison with other chaotic} spread spectrum communication methods

In the following, the traditional chaotic binary spread spectrum communication method in Ref. [24,41], the

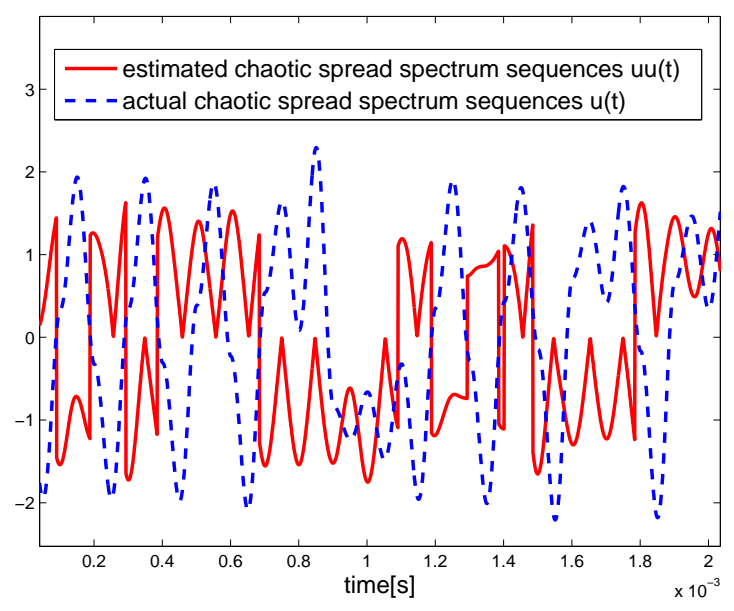

(a)
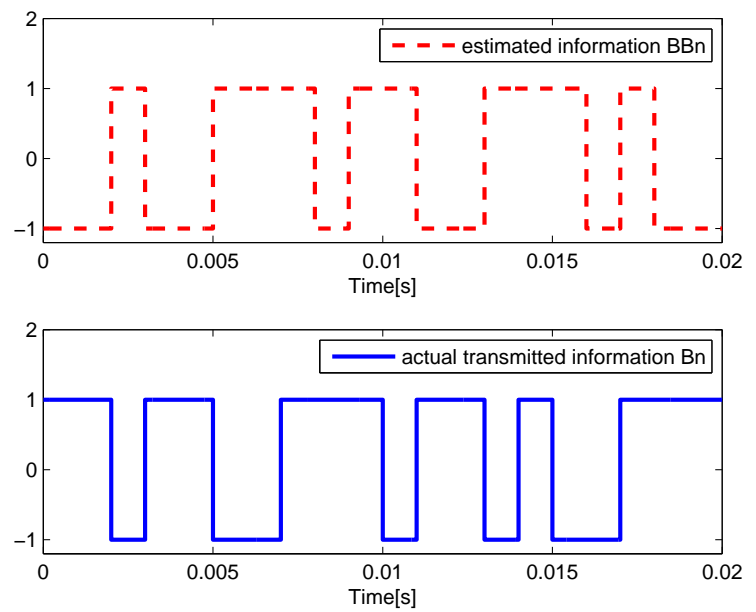

(b)

Fig. 10 (a) Comparison of waveforms of estimated and actual chaotic spread spectrum sequences. (b) Recovered information using method in [35] and actual transmitted information.

chaotic real value spread spectrum communication method in Ref. [30], and the proposed chaotic spread spectrum communication method in this paper are compared using the same acoustic channel parameters given by Table 1 . The communication system configuration and the information transmission rate for the method in Ref. [24], the method in Ref. [30], and the proposed method are given in Table 2. The parameters in Ref. [24] are given as follows: the duration of the probe signal is 0.02 millisecond, the time interval between the probe signal and the transmitted information is 50 millisecond, the duration of the transmitted information is 1.02 second and the time interval between the transmitted information and the next probe signal is 150 millisecond. From Table 2, we can see that, due to 
Table 2 Comparison of different chaotic spread spectrum systems

\begin{tabular}{cccc}
\hline method & method in $[24]$ & method in [30] & proposed method \\
\hline modulation & BPSK & complex modulation & None \\
carrier frequency & $50 \mathrm{kHz}$ & $50 \mathrm{kHz}$ & $50 \mathrm{kHz}$ (base frequency) \\
equalization & PTRM & None & None \\
despreading method & known spread sequence & UKF estimation & synchronous frame \\
symbol transmission rate & $41.802 \mathrm{kbit} / \mathrm{s}$ & & $50 \mathrm{kbit} / \mathrm{s}$ \\
information transmission rate & $836.050 \mathrm{bit} / \mathrm{s}$ & $1000 \mathrm{bit} / \mathrm{s}$ & $980.39 \mathrm{bit} / \mathrm{s}$ \\
\hline
\end{tabular}

adopting Passive Time Reversal Mirror (PTRM) equalization and the requirement to send the probe signal, the transmission rate for the method in Ref. [24] is low. But the bit transmission rate of the three methods are comparable.

In the following, we check the BER of the three methods. We obtain BER curves versus the SNR for the method in Ref. [24] by using different spread spectrum codes, for the method in Ref. [30], and for our proposed method, respectively. Figure 11(a) shows the BER versus SNR for all these different methods using the same communication channel configuration. From Fig. 11(a), we learn that the BER of the method in Ref. [30] is highest and the BER of the proposed method is lowest.

5.7 Comparison with differential chaos shift keying (DCSK)

DCSK is a method to send a piece of the chaotic signal as a reference signal and then the corresponding piece of the chaotic signal modulated with information. The reference signal has a similar function as the synchronization frame in our proposed method. But these two methods are different in the following points: Firstly, the reference signal of DCSK has the same duration as the information bearing signal. But in our proposed method, only a smaller portion of duration of spread spectrum signal is used as the synchronization frame. This point has an important effect on the bit transmission rate and the bandwidth utilization rate, the bit transmission rate of our proposed method is $R_{p}=\frac{f}{L+1}$, where $f$ is the base frequency of the hybrid system and $L$ is the spread spectrum gain. While the bit transmission rate of the DCSK is $R_{D}=\frac{f_{s}}{2 L}$, where $f_{s}$ is the sampling frequency in the traditional DCSK communication system. Assume a communication channel bandwidth to be $B(H z)$. Then, the bandwidth utilization rates of our proposed method and DCSK are $\frac{f}{(L+1) B}$ and $\frac{f_{s}}{2 B L}$, respectively. Secondly, our

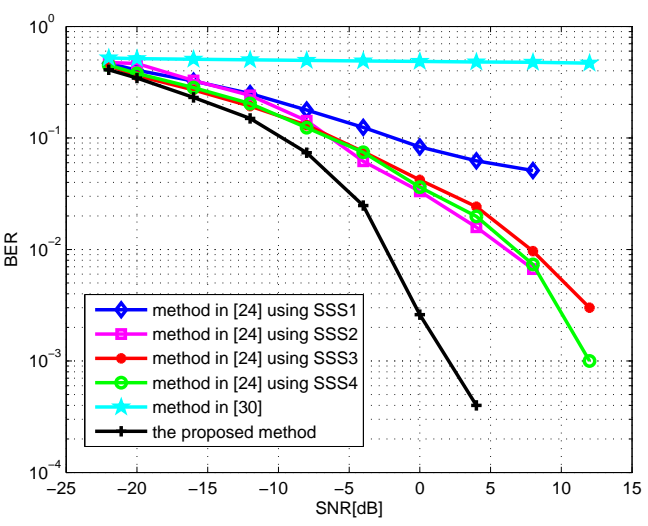

(a)

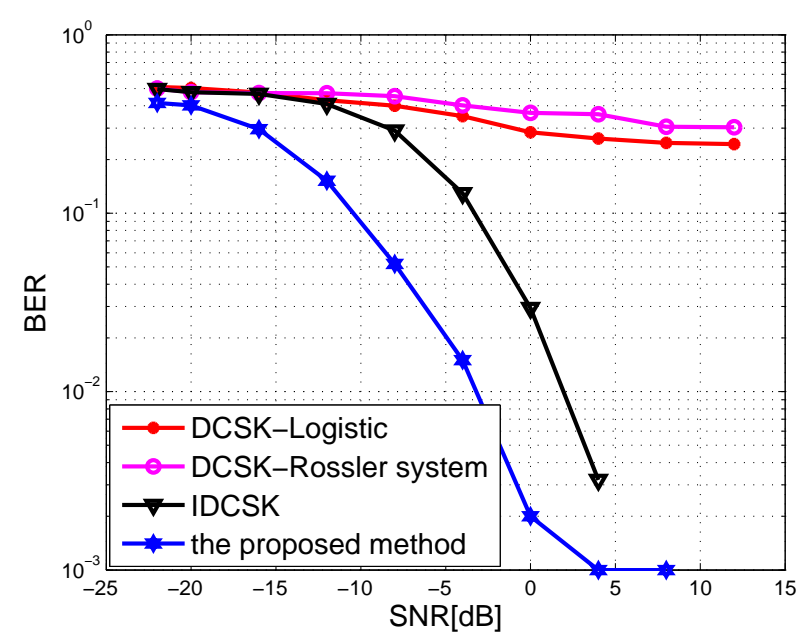

(b)

Fig. 11 (a) BER of different chaotic spread spectrum systems in the same acoustic channel. (b) BER of DCSK and the proposed method in the acoustic channel.

proposed method uses a special chaotic signal and it$\mathrm{s}$ corresponding match filter to decrease the effect of noise and interference, which helps to get better performance in the complicated communication channel, such as under water acoustic channel. To show this point, 
the BER comparison with DCSK using logistic map, DCSK using rossler chaotic signal, an improved differential chaos-shift keying (I-DCSK) system [42][43], and the proposed method are given in Fig. 11(b). In Fig. 11(b), $L=63, f=f_{s}=30 k H z$, the bit transmission rate and bandwidth utilization rate in this case are given in Table 3. We can see the proposed method has lower BER. From Table 3 and Fig. 11(b), we see that our proposed method is superior to the DCSK and the I-DCSK in the underwater communication channel.

A point to be noticed here is that we also use other acoustic channel parameters to test the result in Subsections 5.6 and 5.7, and get similar results, which are not described here for the limited length of the paper.

\section{Conclusions}

In this paper, a novel chaotic direct sequence spread spectrum system for underwater acoustic communication is proposed, which is considerably different from the conventional PN spread spectrum methods, the chaotic real value spread spectrum methods and DCSK. It has the following features:

(1) The proposed method uses the real-value chaotic signal as a spread spectrum sequence. Therefore, it is similar to that of the CRSS(CD3S) methods, however, the continuous chaotic signal generated by the hybrid system includes the discrete state like PN code in conventional CBSS method. This method does not need the channel modulation and demodulation, which are necessary for the CRSS method in Ref. [30]. Therefore, it simplifies the communication system configuration. In addition, this method does not need to perform quantization, as needed by the CBSS method in Ref. [24], which avoids the periodicity of the spread spectrum sequence caused by the quantization.

(2) From Ref. [2], we know that the underwater channel distorts the chaotic signal, but the signal remains preserved with all its important topological structure, and the proposed method uses the match filter to decrease the effect of noise and inter-symbol interference caused by multipath propagation. The match filter replaces the conventional channel equalization techniques with a simpler system configuration. This helps to get a low BER.

(3) The proposed method uses a synchronous frame for ease demodulation, and it is very simple as compared to the conventional CBSS, which needs to know the precise original spread spectrum sequence at the receiver end or the exact synchronization between the transmitter and the receiver, as that in Ref. [24]. As compared to the method in Ref. [30], the proposed method does not use filter algorithm to estimate the information and spread spectrum sequence, hence, the performance is independent of noise properties. The synchronization frame is much shorter as compared to the reference signal of the conventional DCSK, which helps to get higher bit transmission rate and bandwidth utilization rate.

(4) The proposed method has comparable bit transmission rate to the conventional CBSS or CRSS, as can be seen from Table 2 .

(5) Other merits of the proposed method include anti-jamming, resistance to Doppler effect, environmental amity in the sense of not disturbing the whale's navigation by using noise like chaotic signal.

The proposed method shows a great potential for under water communication applications. The future work is to test the proposed method in a lake or sea trial.

Acknowledgement The work is supported in part by NSFC (Grant no. 61172070), IRT of Shaanxi Province (2013KCT-04), EPSRC (Grant no.Ep/1032606/1), Chao Bai was supported by Excellent Ph.D. research fund (310-252071603) at XAUT.

\section{References}

1. J. Heidemann, M. Stojanovic and M. Zorzi, Underwater Sensor Networks: Applications, Advances and Challenges, Phil. Trans. R. Soc. A., 370, 158-175(2012).

2. H.P. Ren, M.S. Baptista, C. Grebogi, Wireless Communication with Chaos, Phys. Rev. Lett., 110(18), 184101(2013).

3. H.P. Ren, M.S. Baptista, C. Grebogi, Uncovering missing symbols in Communication with filtered chaotic signal, Int. J Bifurcat. Chaos, 22(8), 1250119(2012).

4. S.J. Li, Y.Q. Mao and W.D. Peng, An Optimized Selection Algorithm via Adaptive Correlation Threshold on Integrated Chaotic Spread-spectrum Sequences, Proc. IEEE Int. Conf. Wireless Communications and Signal Processing (WCSP), Nanjing, 1-5(2011).

5. L.Y. Liu, X. Yan, H.D. Luo and K. Wang, A Novel Composite Chaotic Sequence for Low Voltage Power Line Spread Spectrum Communication, Proc. of 2012 Power Engineering and Automation Conference (PEAM), Wuhan, 1-6(2012).

6. C.H Chen and Y.W. Hu, A Novel Method of Designing Chaotic Spread Spectrum Sequence Based on Combined Tent-map, Proc. IEEE Microwave Conf., Hangzhou, 14(2011).

7. Gulick D. Encounters with chaos. New York: McGrawHill(1992).

8. R. Nawkhare, A. Tripathi and P. Pokle, DS-SS Communication System Using Pseudo Chaotic Sequences Generator, 2013 IEEE Int. Conf. Communication Systems and Network Technologies (CSNT) Gwalior, 78-82(2013).

9. D.S. Swami and K.K. Sarma, A Logistic Map Based PN Sequence Generator for Direct-Sequence SpreadSpectrum Modulation System, IEEE Int. Conf. Signal Processing and Integrated Networks (SPIN), Noida, 780784(2014). 
Table 3 Comparision of DCSK and chaotic spread spectrum communication system

\begin{tabular}{cccc}
\hline & DCSK & I-DCSK & proposed method \\
\hline chaotic signal used & Logistic map, Rössler system & Logistic map & hybrid dynamical system \\
reference signal length & full length & mixed with data carrier & a little part of spread spectrum signal \\
bit transmission rate & $238.09 b i t / s$ & $476.19 b i t / s$ & $468.75 b i t / s$ \\
bandwidth utilization rate & 0.0059 & 0.0118 & 0.0117 \\
\hline
\end{tabular}

10. H.M. Xie and B.P Wang, A New Piecewise Chaotic Mapping and Its Application in Image Secure Communication, 2012 IEEE 5th Int. Symposium on Parallel Architectures, Algorithms and Programming(PAAP), Taibei, 65-71(2012).

11. H.F Cao, R.X. Zhang and F.L. Yan, Spread Spectrum Communication and Its Circuit Implementation Using Fractional-order Chaotic System via a Single Driving Variable, Commun. Nonlinear SCI., 18(2), 341$350(2013)$.

12. W.J. He, L.Y. Bao and D.F. Zhao, The Analyses of an Improved 2-order Chebyshev Chaotic Sequence, 2011 IEEE Int. Conf. Computer Science and Network Technology(ICCSNT), Harbin, 1224-1227(2011).

13. N. Rahnama and S. Talebi, Performance Comparison of Chaotic Spreading Sequences Generated by Two Different Classes of Chaotic Systems in A Chaos-based Directsequence Code Division Multiple Access System, IET Commun., 7(10), 1024-1031(2013).

14. J. Zhang, J. Cheng and G.X. Li, Chaotic Spread Spectrum Sequences Using Chaotic Quantization, 2007 IEEE Int. Symp. Intelligent Signal Processing and Communication Systems(ISPACS), Xiamen, 40-43(2007).

15. C.J. Pen and B. Wang, An Optimal Design of Infinitely Long Period Spreading Sequences Based on Logistic Mapping, 2012 IEEE Symp. ICT and Engergy Efficiency and Workshop on Information Theory and Security(CIICT), Dublin, 147-151(2012).

16. A.J. Michaels and C.C. Lau, Quantization Effects in Digital Chaotic Communication Systems, IEEE Military Communications Conf.(MILCOM), San Diego, 15641569(2013).

17. X.W. Ceng, Spread Spectrum Comunication and Its Multiple Access Technology, Xi'an: Xidian University Press, Xi'an, China(2004).

18. R. Vali, S.M. Berber and S.K. Nguang, Analysis of ChaosBased Code Tracking Using Chaotic Correlation Statistics, IEEE Trans. Circuits Syst. I, Reg. Papers, 59(4), 796-805(2012).

19. R. Vali, S.M. Berber and S.K. Nguang, Accurate Derivation of Chaos-Based Acquisition Performance in A Fading Channel, IEEE Trans. Wireless Commun., 11(2), 722-731(2012).

20. R. Vali, S.M. Berber and Gsmieeee, Using Chip Interleaving to Improve Chaos-based Code Tracking Performance, IEEE 19th Asia-Pacific Conf. Communications(APCC), Denpasar, 283-286(2013).

21. N. Li and Q.B. Tang, Application Study of Spatiotemporal Chaotic Sequence in Code Division Multiple Access Communication System, Advances in Computer Science and Its Application(ACSA), 279, 943-949(2014).

22. S.L. Wang, J. Zhu and J. Zhou, OFDM-based Chaotic Spread Spectrum Communications with High Bandwidth Efficiency, IEEE Int. Conf. Control Engineer- ing and Communication Technology(ICCECT), Liaoning, 940-943(2012).

23. D. Li and F.B. Jiang, Design of The DS-UWB Communication System based on Chaotic Variable Spreading Factor, IEEE 7th Int. Conf. Computational Intelligence and Security(CIS), Hainan, 717-720(2011).

24. L.H. Lei, F. Xu, A Chaotic Direct-Sequence SpreadSpectrum Communication System in Shallow Water, IEEE Int. Conf. Control, Automation and Systems Engineering(CASE), Singapore, 1-4(2011).

25. H.P. Ren, Q.J. Kong, C. Bai, A Chaotic Spread Spectrum System for Underwater Acoustic Communication, IEEE Int. Conf. Wireless Symposium, Shenzhen, 1-4(2015).

26. C. Laot, P. Coince, Experimental Results on Adaptive MMSE Turbo Equalization in Shallow Underwater Acoustic Communication, 2010 IEEE Oceans, Sydney, NSW, Australia, 1-5(2010).

27. T.Y. Sui, Z.K. Yuan and C.H. Zhang, A Coherent Underwater Acoustic Communication System Based on Joint Iterative Equalization and Decoding Algorithm, 2010 IEEE Youth Conf. Information Computing and Telecommunications(YC-ICT), Beijing, 73-76(2010).

28. U. Parlitz and S. Ergezinger, Robust Communication Based on Chaotic Spreading Sequences, Phys. Lett. A, 188(2), 146-150(1994).

29. H.B. Ghobad and C.D. McGillem, A Chaotic DirectSequence Spread-Spectrum Communication System, IEEE Trans. Commun., 42(234), 1524-1527(1994).

30. S. Azou, G. Burel and C. Pistre, A Chaotic DirectSequence Spread-Spectrum System for Underwater Communication, 2002 IEEE Oceans, Biloxi, Mississippi, USA, 2409-2415(2002).

31. S. Azou and G. Burel, Design of A Chaos-based Spreadspectrum Communication System Using Dual Unscented Kalman Filters, 2002 IEEE Communications, Bucharest, Romania, 1-6(2002).

32. S. Azou, C. Pistre and L. Le Duff, Sea Trial Results of A Chaotic Direct-sequence Spread Spectrum Underwater Communication System, 2003 IEEE Oceans, San Diego, CA, USA, 1539-1546(2003).

33. M. Luca, S. Azou, G. Burel and A. Serbanescu, A Complete Receiver Solution for A Chaotic Direct-Sequence Spread-Spectrum Communication System, IEEE Int. Symp. Circuits and Systems, 3813-3816(2005).

34. S. Azou, G. Burel and A. Serbanescu, Multipath Combing in Chaotic Direct-Sequence Spread Spectrum Communications Through Dual Estimation, 2005 IEEE Oceans, Europe, 616-621(2005).

35. J.F. Hu and J.B. Guo, Breaking A Chaotic Secure Communication Scheme, Chaos: An Interdisciplinary Journal of Nonlinear Science, 18(1), 029901(2008).

36. G. Lu and X. Bo, Breaking A Chaotic Direct Sequence Spread Spectrum Communication System Using Interacting Multiple Model-unscented Kalman filter, Chaos:An 
Interdisciplinary Journal of Nonlinear Science, 22(1), 043122(2012).

37. X.Z. Xu and J.B. Guo, Combined Equalization and Demodulation of Chaotic Direct Sequence Spread Spectrum Signals for Multipath Channels, Circuits, Systems and Signal Process, 32(6), 2957-2969(2013).

38. T. Li et al., Blind Demodulation of Chaotic Direct Sequence Spread Spectrum Signals Based on Particle Filters, Entropy, 15, 3877-3891(2013).

39. M. Chitre, A High-frequency Warm Shallow Water Acoustic Communications Channel Model and Measurements, J. Acoust. Soc. Am., 122(5), 2580-2586(2007).

40. N.J. Corron, J.N. Blakely and M.T. Stahl, A Matched Filter for Chaos, Chaos: An Interdisciplinary Journal of Nonlinear Science, 20(1), 023123(2010).

41. G. L. stüber, Principles of mobile communication, Springer, Third Edition, (2005).

42. G. Kaddoum, Wireless Chaos-Based Communication Systems: A Comprehensive Survey, IEEE Access, 4, 2621-2648(2016)

43. G. Kaddoum, E. Soujeri, C. Arcila, and K. Eshteiwi, IDCSK: An improved noncoherent communication system architecture, IEEE Trans. Circuits Syst. II, 62(9), 901905(2015). 